\title{
PREVER A EVOLUÇÃO DOS MERCADOS DE AÇÕES EM TEMPO DE CRISE: O CASO DA EURONEXT LISBON
}

FORECASTING THE EVOLUTION OF STOCK MARKETS IN TIMES OF CRISIS: THE CASE OF EURONEXT LISBON PREDECIR LA EVOLUCIÓN DE LOS MERCADOS DE ACCIONES EN TIEMPO DE CRISIS: EL CASO DE EURONEXT LISBON

Júlio Lobão (ilobao@fep.up.pt)

Daniela Rocha (danielarocha.mrg@gmail.com) ${ }^{\star \star}$

\section{RESUMO}

Neste artigo estudamos a utilidade da análise fundamental para prever a evolução do preço das ações cotadas na Euronext Lisbon no contexto da crise financeira global pós-2007. Na elaboração das estratégias de investimento foi considerado um conjunto alargado de 49 indicadores financeiros, aplicados a 29 ações. Contrariamente à generalidade dos estudos sobre o mesmo tema, as estratégias de investimento fundamentais analisadas não se mostraram lucrativas. Os resultados obtidos sugerem que o aumento da incerteza provocado por eventos como crises financeiras diminui a utilidade das estratégias baseadas no recurso a indicadores financeiros.

Palavras-chave: análise fundamental, eficiência de mercado, mercado de ações, crise financeira, Portugal.

\begin{abstract}
In this article we study the usefulness of fundamental analysis to forecast the prices of stocks listed on Euronext Lisbon in the context of the post-2007 global financial crisis. In the design of the investment strategies, a broad set of 49 financial indicators were considered, applied to 29 different stocks. In contrast to most studies on the same subject, the fundamental investment strategies were not found to be profitable. This result suggests that the increased uncertainty caused by events such as thefinancial crisis diminishes the usefulness of strategies based on the use of financial indicators.
\end{abstract}

Keywords: fundamental analysis, market efficiency, stock market, financial crisis, Portugal.

\section{RESUMEN}

En este artículo se estudia la utilidad del análisis fundamental para predecir la evolución del precio de las acciones que cotizan en Euronext Lisboa en el contexto de la crisis financiera global post-2007. En la elaboración de las estrategias de inversión se consideró una amplia gama de 49 indicadores financieros aplicados a 29 acciones. Al contrario de la mayoría de los estudios sobre el mismo tema, las estrategias de inversión fundamentales analizados no fueron rentables. Los resultados sugieren que el aumento de la incertidumbre causada por acontecimientos como una crisis financiera disminuye la utilidad de las estrategias basadas en el uso de los indicadores financieros.

Palabras-llave: análisis fundamental, eficiencia del mercado, mercado de acciones, crisis financiera, Portugal. 


\section{Eqitania}

s c i e $\Omega$ c i a

* Investigador do CEPESE - Centro de Estudos de População, Economia e Sociedade; Professor auxiliar na Faculdade de Economia do Porto, Universidade do Porto.

** Faculdade de Economia do Porto, Universidade do Porto.

Submitted: 13th July 2015

Accepted: 13th November 2015 


\section{INTRODUÇÃO}

De acordo com a versão semi-forte da hipótese de eficiência de mercado (Fama, 1970), a informação pública disponível como, por exemplo, a informação contabilística não deveria poder ser usada para detetar, com sucesso, ativos financeiros sub ou sobreavaliados. Por conseguinte, qualquer estratégia concebida com base em informação financeira de acesso público não deveria proporcionar rendibilidades supranormais de forma sistemática.

Em contraste com este argumento, os analistas fundamentais acreditam que pode haver erros de preço nos mercados financeiros no curto prazo, admitindo-se que o preço correto será atingido apenas no longo prazo. Nesta perspetiva, poderão ser obtidas rendibilidades supranormais através da compra (venda) de ativos subavaliados (sobreavaliados). Na identificação dos ativos sub e sobreavaliados, os analistas fundamentais fazem uso de indicadores contabilísticos e financeiros relativos ao desempenho das empresas emitentes dos ativos.

Neste artigo testamos a utilidade dos indicadores financeiros na previsão das rendibilidades das ações cotadas na Euronext Lisbon durante o período da crise financeira de 2008. A motivação para o estudo advém de dois resultados bem estabelecidos na literatura. Em primeiro lugar, a evidência empírica conclui que a análise fundamental, com recurso a indicadores financeiros, é geralmente útil na previsão da evolução do preço das ações permitindo obter ganhos de forma consistente (e.g., Holthausen e Larcker, 1992; Charitou e Panagiotides, 1999; Alexakis et al., 2010; Hyde, 2014). Mas, por outro lado, constata-se que o nível de incerteza aumenta de forma significativa em períodos de crise financeira. $O$ aumento da incerteza torna mais difícil prever a evolução dos mercados financeiros em resultado, entre outros fatores, do aumento da aversão ao risco dos investidores, da maior dificuldade na difusão da informação nos mercados e da maior divergência nas previsões dos agentes económicos (Baker et al., 2012; Bloom, 2014; Orlik e Veldkamp, 2014).

Atendendo a estes dois resultados, é então útil recorrer a indicadores financeiros para prever a evolução dos preços das ações num período de crise financeira? É a esta pergunta a que procuramos dar resposta.

Para isso, consideramos um vasto conjunto de indicadores financeiros na previsão das rendibilidades das ações cotadas na Euronext Lisbon. Utilizamos um modelo logístico com dados pré-crise (2004-2007) para integrar todos os indicadores e para prever as ações com maior probabilidade de apresentarem rendibilidades supranormais positivas e negativas, para diferentes períodos temporais, durante o período de crise financeira (2008-2011). Essas previsões são utilizadas para levar a cabo estratégias de investimento baseadas na assunção de posições longas nas ações com maior probabilidade de obtenção de rendibilidades positivas e de posições curtas nas ações com maior probabilidade de obtenção de rendibilidades negativas. 


\section{Egitania}

$s$ c i e $\Omega$ c i a

O estudo da utilidade da informação financeira num mercado de reduzida dimensão como a Euronext Lisbon e durante um período de crise constitui uma contribuição relevante para a literatura por diversas razões. Em primeiro lugar, os estudos existentes sobre esta temática têm incidido, na sua grande maioria, sobre os mercados dos EUA e do Reino Unido. Ora, as economias destes dois países são exemplos de sucesso invulgar na sua trajetória de desenvolvimento ao longo do último século pelo que as conclusões aí obtidas não são necessariamente extrapoláveis para os mercados financeiros dos restantes países. Esta linha de raciocínio tem sido confirmada por diversos estudos empíricos que concluem que as especificidades de cada país são fatores relevantes nas escolhas dos investidores e na formação dos preços de mercado (e.g., Beckman et al., 2008; Chui et al., 2010). No caso da Euronext Lisbon, existem igualmente estudos que apontam para a especificidade desse mercado tanto ao nível dos comportamentos dos investidores como da formação dos preços (e.g., Lobão e Serra, 2007; Lobão e Lopes, 2014). Em segundo lugar, o presente estudo debruça-se sobre as capacidades preditivas da informação financeira no período da crise financeira internacional, que se iniciou com a crise do subprime nos EUA em 2007 (Crouhy et al., 2008). Este estudo é, tanto quanto é do nosso conhecimento, um dos primeiros acerca desta temática no período de crise referido. Em terceiro lugar, o interesse do estudo da Euronext Lisbon decorre do facto dos resultados obtidos nos estudos existentes, apesar de apontarem, em geral, para a utilidade da informação financeira, parecerem depender do mercado analisado e do período temporal sob análise (ver secção seguinte). Por conseguinte, são necessárias mais evidências obtidas em mercados e períodos temporais distintos para se chegar a conclusões mais robustas acerca da lucratividade potencial das estratégias de investimento a que nos temos vindo a referir. Em quarto lugar, os resultados obtidos, quando conjugados com a evidência existente para outros mercados, podem ser úteis na avaliação dos determinantes das rendibilidades das ações usadas nos modelos empíricos existentes. Nesta medida, os resultados podem levar a um melhor entendimento do comportamento dos preços das ações e, por conseguinte, também a uma melhor compreensão das decisões de investimento financeiro. Por isso, os resultados deste estudo são também relevantes para os analistas fundamentais. Estes agentes têm diante si a difícil tarefa de escolher, de entre os numerosos indicadores financeiros ao seu dispor, aqueles que supõem serem mais úteis na previsão das rendibilidades futuras das ações. Este estudo contribuirá para identificar esses indicadores financeiros.

O artigo organiza-se em seis secções. Na secção seguinte revê-se a literatura relevante para o estudo levado a cabo. Na terceira secção são apresentados os dados e os métodos do estudo empírico. Na quarta secção discutem-se os resultados obtidos na estimativa do modelo logístico. Na secção 5 apresentam-se os resultados referentes à aplicação das estratégias de investimento baseadas no modelo estimado. A sexta secção contém as principais conclusões do estudo. 


\section{Egitania}

s c i e $\Omega$ c i a

\section{REVISÃO DE LITERATURA}

Foram vários os autores que se debruçaram sobre a utilidade da informação fundamental. Os estudos nesta área iniciaram-se com Ou e Penman (1989) e Holthausen e Larcker (1992).

Ou e Penman (1989) partiram de um conjunto de 68 indicadores financeiros, selecionados com base na sua disponibilidade. De seguida, foram utilizados apenas os indicadores estatisticamente significativos (34 indicadores dos 68 inicialmente considerados) para calcular uma medida sintética, denominada por Pr, para prever a evolução futura dos resultados das empresas cotadas na NYSE e na AMEX no período 1973-1983. A partir dessa medida foram definidas posições longas (em empresas com $\operatorname{Pr}>=0,60$ ) e posições curtas (em empresas com $\operatorname{Pr}<=0,40)$, de dimensão semelhante, nas ações das empresas. A estratégia assim definida gerou uma rendibilidade supranormal de 14,53\% no período de investimento de 24 meses.

Holthausen e Larcker (1992) consideraram os indicadores sugeridos por Ou e Penman (1989) para estudar a relação entre a informação contabilística e as rendibilidades das ações durante o período 1978-1988. As estratégias de investimento, neste caso, proporcionaram rendibilidades inferiores às encontradas por Ou e Penman (1989), mas ainda assim significativamente diferentes de zero; para um período de investimento de 12 meses, gerou-se uma rendibilidade supranormal entre 4,26\% e 7,97\% dependendo da medida usada.

Setiono e Strong (1998) aplicaram os métodos propostos por Ou e Penman (1989) e por Holthausen e Larcker (1992) ao mercado do Reino Unido no período 1971-1988. Os resultados evidenciam que um investidor desse país poderia ter obtido uma rendibilidade supranormal de 17,38\% ou de 5\% num investimento ao longo de 24 meses se a estratégia proposta por Ou e Penman (1989) ou por Holthausen e Larcker (1992), respectivamente, fosse a adotada.

Charitou e Panagiotides (1999) corroboram os resultados de Setiono e Strong (1998) para o Reino Unido para o período 1991-1995: a informação financeira permitiria prever os resultados do ano seguinte e as estratégias de investimento em ações baseadas na variável Pr permitiriam obter rendibilidades supranormais positivas. Mohanram (2005) aplica uma estratégia de investimento baseada em indicadores contabilísticos ao mercado dos EUA para distinguir entre ações "vencedoras" e "perdedoras" no universo das ações com reduzido book-to-market. A estratégia permite obter rendibilidades supranormais significativas no período 1979-1999.

Os resultados obtidos nos estudos existentes para os EUA e para o Reino Unido, apesar de sugerirem que os indicadores financeiros contêm informação útil acerca da rendibilidade futura das ações, parecem ser algo sensíveis ao período temporal e mercados considerados. Por exemplo, Holthausen e Larcker (1992) referem que a sua estratégia foi mais lucrativa na subamostra entre 1978 e 1982 do que na sub-amostra entre 1983 e 1988 enquanto que no caso de Ou e Penman (1989), a estratégia apenas se mostrou lucrativa no primeiro sub-período. Além disso, Holthausen e Larcker (1992) mostram que a estratégia de Ou e Penman (1989) produziria melhores resultados nas ações cotadas na NYSE/AMEX do que nas ações transacionadas no mercado OTC. Mais: como vimos, Setiono e Strong (1998) concluíram que 


\section{Egitania}

$s$ c i e $\cap$ c i a

a estratégia proposta por Ou e Penman (1989) seria bem-sucedida no Reino Unido enquanto que a estratégia de Holthausen e Larcker (1992) não produziria resultados significativos nesse mercado.

Alguns dos artigos na matéria debruçam-se sobre os mercados europeus. É o caso do estudo de Martinez (1999), por exemplo, que mostrou que os rácios financeiros ajudam a prever as rendibilidades das ações de 50 empresas transacionadas em França. E, mais recentemente, Alexakis et al. (2010) concluem que as informações relativas à liquidez da empresa e à sua solvabilidade permitem conceber estratégias de investimento lucrativas nas ações listadas na bolsa grega.

Os mercados asiáticos têm vindo a ser igualmente analisados. Por exemplo, Cheung et al. (1997) estudaram a utilidade do price-earnings ratio e do book-to-market na previsão das rendibilidades das ações de uma amostra de empresas sedeadas em Hong-Kong. A conclusão é que esses indicadores são úteis para prever as rendibilidades das ações até um horizonte de dois anos. Chung e Kim (2001) estudaram a questão no mercado de ações sul-coreano tendo encontrado rendibilidades supranormais entre 11,44\% e 16,92\% para um horizonte de investimento de 12 meses. Kang e Ding (2005) corroboram os resultados de Chung e Ding (2005) noutros mercados asiáticos. Num estudo relacionado, Avkiran e Morita (2010) socorremse de rácios financeiros para prever, com sucesso, a evolução dos preços das ações das instituições bancárias japonesas.

Mais recentemente, as investigações na área têm-se alargado a outros mercados. Por exemplo, Abekah (2005) e Tantipanichkul (2011) concluem a favor da utilidade da informação financeira considerando as ações transacionadas nos mercados do Gana e Tailândia, respetivamente. Por último, Hyde (2014) estuda os mercados que fazem parte do MSCI Emerging Markets Index. O autor analisa o poder das estratégias fundamentais no período 2000-2011 para concluir que os indicadores contabilísticos são úteis para a previsão das rendibilidades das ações.

O estudo acerca da utilidade da informação contabilística é especialmente útil em períodos de crise como o abordado no presente artigo. De facto, existem indicações na literatura de que, em períodos de maior volatilidade, fatores como o sentimento de mercado ou os enviesamentos cognitivos dos investidores podem substituir-se aos fundamentais e influenciar, de forma decisiva, a evolução do preço dos ativos financeiros (Kahneman et al., 1982; Shiller, 2000).

Em resumo, da revisão da literatura pode-se concluir que os resultados, tanto no caso dos mercados mais desenvolvidos como no caso dos mercados emergentes, apontam genericamente para a utilidade da informação contabilística na previsão da evolução dos preços das ações. No nosso estudo, complementamos a evidência existente acerca da utilidade da informação fundamental levando a cabo testes sobre essa matéria num outro mercado, ainda não estudado, o Euronext Lisbon. 


\section{DADOS E MÉTODOS DO ESTUDO EMPÍRICO}

\subsection{DADOS}

Foram inicialmente considerados os 68 indicadores utilizados por Ou e Penman (1989). No entanto, foi necessário excluir 19 desses indicadores por existir um número considerável de dados em falta no período da amostra. Foram assim utilizados os 49 indicadores financeiros que se indicam na tabela 1, a seguir:

\begin{tabular}{|c|c|c|}
\hline 1. Índice de Liquidez Corrente & 18. $\Delta$ em 17 & 35. Vendas / Dívidas de terceiros \\
\hline 2. $\Delta \%$ em 1 & 19. Dívida / Capital Próprio & 36. Vendas / Inventários \\
\hline 3. Índice de Liquidez Reduzida & 20. $\Delta \%$ em 19 & 37. $\Delta \%$ em 36 \\
\hline 4. $\Delta \%$ em 3 & $\begin{array}{l}\text { 21. Dívida de Longo Prazo / Capital } \\
\text { Próprio }\end{array}$ & 38. Fundo de maneio / Vendas \\
\hline 5. Tempo médio de recebimento & 22. $\Delta \%$ em 21 & 39. $\Delta \%$ em 38 \\
\hline 6. $\Delta \%$ em 5 & 23. Capital Próprio / Imobilizado & 40. Vendas / Imobilizado \\
\hline $\begin{array}{l}\text { 7. Tempo médio da rotação de } \\
\text { inventários }\end{array}$ & 24. $\Delta \%$ em 23 & 41. $\Delta \%$ Total Ativo \\
\hline 8. $\Delta \%$ em 7 & 25. Vendas / Total Ativo & 42. Fluxos financeiros / Passivo \\
\hline 9. Inventários / Ativo & 26. $\Delta \%$ em 25 & 43. Fundo de maneio / Ativo \\
\hline 10. $\Delta \%$ em 9 & 27. Rendibilidade do Ativo & 44. $\Delta \%$ em 43 \\
\hline 11. $\Delta \%$ Inventários & 28. EBITDA / Vendas & 45. Resultados Operacionais / Ativo \\
\hline 12. $\Delta \%$ Vendas & 29. $\Delta \%$ em 28 & 46. $\Delta \%$ em 45 \\
\hline 13. $\Delta \%$ Amortizações & 30. Resultados antes de impostos & $\begin{array}{l}47 . \Delta \% \text { Passivo exigível a médio e longo } \\
\text { prazo }\end{array}$ \\
\hline 14. $\Delta$ Dividendo por acção & $31 . \Delta \%$ em 30 & 48. $\Delta \%$ Fundo de maneio \\
\hline $\begin{array}{l}\text { 15. Amortizações / (Imobilizado } \\
\text { corpóreo + Imobilizado incorpóreo) }\end{array}$ & 32. Resultados Líquidos/Vendas & $\begin{array}{l}\text { 49. Resultados Líquidos/Fluxos de } \\
\text { Caixa }\end{array}$ \\
\hline 16. $\Delta \%$ em 15 & 33. $\Delta \%$ em 32 & \\
\hline 17. Rendibilidade Financeira & $\begin{array}{l}\text { 34. Vendas / (Depósitos Bancários + } \\
\text { Caixa) }\end{array}$ & \\
\hline
\end{tabular}

Tabela 1 - Indicadores financeiros selecionados para o estudo

Os dados contabilísticos e financeiros recolhidos têm frequência anual e dizem respeito ao período entre os anos de 2004 e 2011. A informação necessária para o cálculo dos indicadores foi recolhida na base de Dados SABI van Dijke e os dados em falta foram recolhidos diretamente dos balanços e demonstrações de resultados publicados pela Comissão de Mercado de Valores Mobiliários (CMVM). 


\section{Egitania}

$s$ c i e $\Omega$ c i a

O estudo incide sobre as ações cotadas na Euronext Lisbon. Uma vez que é necessário, na seleção dos títulos a analisar, assegurar um nível mínimo de liquidez (e.g., Frankel e Lee, 1998; Beneish et al., 2001), foram selecionadas as ações pertencentes àquele mercado que foram transacionadas em todas as sessões, desde maio de 2005 a abril de 2013. Tal como foi realizado em estudos semelhantes (e.g., Ou e Penman, 1989), foram excluídas da amostra as empresas financeiras e também as empresas de sociedades desportivas por adotarem uma contabilidade específica. Este procedimento conduziu à seleção das 29 ações. Os dados de mercado referem-se às rendibilidades diárias de todas as ações no período já referido e provêm da base de dados Datastream.

\subsection{MODELO LOGÍSTICO COM RENDIBILIDADES SUPRANORMAIS}

O modelo logístico a estimar apresenta como variáveis independentes os indicadores financeiros a que já fizemos menção. A variável dependente é calculada a partir da rendibilidade supranormal das ações. No cálculo da rendibilidade supranormal das ações considerou-se o método de ajustamento ao mercado:

$$
M A R_{i m}=\prod_{t=1}^{m}\left(1+R_{i t}\right)-\coprod_{t=1}^{m}\left(1+R_{M t}\right)
$$

MARim representa a rendibilidade ajustada ao mercado da ação i entre o mês inicial e o mês m. RMt é a rendibilidade de mercado e Rit é a rendibilidade da ação i. Como proxy da rendibilidade de mercado adotou-se o Portuguese Stock Index General (PSI Geral).

Uma vez que se pretende estudar o impacto nos preços de mercado da informação financeira com divulgação pública, é necessário atender ao momento da divulgação da informação. Dado que as empresas selecionadas têm como fim do ano fiscal o mês de dezembro e visto o Código das Sociedades Comerciais em vigor em Portugal referir que as informações financeiras devem ser prestadas, o mais tardar, 5 meses após o ano fiscal (artigo 65․ Do Código das Sociedades Comerciais), optamos por calcular as rendibilidades anuais com início em maio de cada ano e final em abril do ano seguinte.

A relação entre os 49 indicadores financeiros e as rendibilidades ajustadas ao mercado será assegurada através de um modelo logístico. Na estimativa deste modelo é utilizada uma variável latente $Y^{\star}$, sendo que:

$$
Y_{i}=\left\{\begin{array}{l}
1, \text { se } Y_{i}^{*}>0 \\
0, \text { se } Y_{i}^{*} \leq 0
\end{array}\right.
$$

Yi é uma variável discreta observável. Assim, a regressão logística a estimar terá como variáveis independentes os 49 indicadores financeiros anuais apresentados na tabela 1 e como variável dependente a variável binária Yi que assume o valor nulo se a rendibilidade supranormal da ação i for negativa e o valor unitário se for positiva. A equação a estimar é então a seguinte:

$$
Y_{i}=c+B_{1} \operatorname{Ind} 1+B_{2} \operatorname{Ind} 2+B_{3} \operatorname{Ind} 3+\ldots+B_{49} \operatorname{Ind} 49
$$


Uma vez que um dos nossos objetivos é o estudo da utilidade da aplicação, em períodos de crise, da informação financeira obtida em períodos de não-crise, consideramos para a estimativa do modelo os dados referentes à Euronext Lisbon no período 2004-2007. No cálculo da variável binária descrita anteriormente foram utilizadas as rendibilidades supranormais com periodicidade anual, no período subsequente a cada um desses anos. Assim, por exemplo, para uma dada empresa e para os indicadores financeiros referidos a dezembro de 2004, o valor da variável binária decorre do sinal da rendibilidade supranormal com início no mês de maio de 2005 e o fim em abril de 2006.

Em resumo, pretende-se utilizar o modelo logístico para prever as probabilidades das rendibilidades supranormais serem positivas $(y=1)$ ou negativas $(y=0)$. Nessa estimativa recorrese aos indicadores financeiros históricos das respetivas empresas.

\section{ESTIMATIVA E CAPACIDADE PREDITIVA DO MODELO LOGÍSTICO}

Na estimativa da regressão logística são consideradas um total de 116 observações, sendo que em 48 dessas observações a variável binária assume o valor unitário e nas restantes 68 observações assume o valor nulo. Os coeficientes estimados e os respetivos p-values para os 49 indicadores são apresentados na tabela 2, a seguir.

\begin{tabular}{|c|c|c|}
\hline Variável & Coeficiente & $p$-value \\
\hline C & $-7,097514$ & 0,2441 \\
\hline IND1 & $-16,662820$ & 0,0959 \\
\hline IND2 & 5,412478 & 0,5591 \\
\hline IND3 & 11,502420 & 0,1653 \\
\hline IND4 & $-4,021331$ & 0,5918 \\
\hline IND5 & $-0,003388$ & 0,5369 \\
\hline IND6 & $-2,944339$ & 0,1841 \\
\hline IND7 & 0,012728 & 0,0902 \\
\hline IND8 & 0,513792 & 0,844 \\
\hline IND9 & 32,704840 & 0,2252 \\
\hline IND10 & 14,589060 & 0,2333 \\
\hline IND11 & $-7,625316$ & 0,5065 \\
\hline IND12 & $-0,000007$ & 0,3416 \\
\hline IND13 & 0,267239 & 0,9397 \\
\hline IND14 & 17,802280 & 0,3278 \\
\hline IND15 & 0,461324 & 0,0089 \\
\hline IND16 & $-3,869190$ & 0,0305 \\
\hline IND17 & 0,000003 & 0,2829 \\
\hline IND18 & 0,092487 & 0,8417 \\
\hline IND19 & 0,063281 & 0,7777 \\
\hline
\end{tabular}

\begin{tabular}{|c|c|c|}
\hline Variável & Coeficiente & $p$-value \\
\hline IND25 & $-0,692590$ & 0,919 \\
\hline IND26 & 1,977584 & 0,0262 \\
\hline IND27 & 16,051640 & 0,287 \\
\hline IND28 & $-0,007996$ & 0,9095 \\
\hline IND29 & 0,255803 & 0,3353 \\
\hline IND30 & 0,000010 & 0,0267 \\
\hline IND31 & $-1,484055$ & 0,0742 \\
\hline IND32 & $-0,021720$ & 0,7397 \\
\hline IND33 & 0,431996 & 0,0833 \\
\hline IND34 & 0,111191 & 0,0022 \\
\hline IND35 & $-0,024243$ & 0,9475 \\
\hline IND36 & $-0,014233$ & 0,9438 \\
\hline IND37 & $-2,377803$ & 0,0151 \\
\hline IND38 & $-0,011968$ & 0,6943 \\
\hline IND39 & $-0,392050$ & 0,0756 \\
\hline IND40 & $-0,000030$ & 0,2205 \\
\hline IND41 & 0,024379 & 0,8758 \\
\hline IND42 & 6,321821 & 0,7652 \\
\hline IND43 & 5,900889 & 0,6534 \\
\hline IND44 & $-2,197748$ & 0,6958 \\
\hline
\end{tabular}




\begin{tabular}{|l|l|l|}
\hline IND20 & 1,377246 & 0,3741 \\
\hline IND21 & 1,165762 & 0,2414 \\
\hline IND22 & $-0,200274$ & 0,5987 \\
\hline IND23 & $-0,000002$ & 0,0235 \\
\hline IND24 & $-0,000020$ & 0,0772 \\
\hline \multicolumn{3}{|c|}{ Número de observações $=116$} \\
R2 de McFadden = 0,628878
\end{tabular}

\begin{tabular}{|c|c|c|}
\hline IND45 & $-30,838380$ & 0,1053 \\
IND46 & $-0,442244$ & 0,037 \\
\hline IND47 & 0,446117 & 0,3118 \\
\hline IND48 & 3,764347 & 0,5021 \\
\hline IND49 & $-0,760596$ & 0,4974 \\
\hline \multicolumn{3}{|c|}{ Estatística RV $=98,95051$} \\
\hline \multicolumn{2}{|c|}{ p-value (estatística RV) $=0,000031$}
\end{tabular}

Tabela 2 - Coeficientes estimados para o modelo logístico e respetivos p-value(dados de 2004 a 2007)

O modelo apresenta-se globalmente significativo ( $\mathrm{p}$-value do rácio de verosimilhança de 0,000031). Como se pode constatar, existem dois indicadores com significância estatística ao nível de 1\%. De fato, as variações positivas nos indicadores 15 (rácio entre amortizações e imobilizado) e 34 (rácio entre vendas e componentes mais líquidas do ativo) contribuem para que as ações respetivas tenham rendibilidades supranormais positivas. Por outro lado, variações positivas nos indicadores 16 (variação no rácio entre amortizações e imobilizado), 23 (rácio entre capital próprio e imobilizado), 37 (variação no rácio entre vendas e inventários) e 46 (variação no rácio entre resultados operacionais e ativo) e uma variação negativa no indicador 26 (variação no rácio entre vendas e ativo) antecipam rendibilidades supranormais negativas nas ações respetivas com um nível de significância estatística de 5\%. Para averiguar da capacidade preditiva do modelo, apresenta-se na tabela 3, a seguir, as taxas de sucesso e insucesso nas previsões para diferentes pontos de quebra.

\begin{tabular}{|c|c|c|c|c|}
\hline & & & \\
\hline & & $y=0$ & $y=1$ & Total \\
\hline \multirow{6}{*}{$\begin{array}{c}\text { Ponto de Quebra } \\
(0,50 ; 0,50)\end{array}$} & $P(y=1)<=0,50$ & 62 & 6 & 68 \\
\hline & $P(y=1)>0,50$ & 6 & 42 & 48 \\
\hline & Total & 68 & 48 & 116 \\
\hline & Corretos & 62 & 42 & 104 \\
\hline & $\%$ Corretos & 91,18 & 87,5 & 89,66 \\
\hline & $\%$ Incorretos & 8,82 & 12,5 & 10,34 \\
\hline \multirow{6}{*}{$\begin{array}{c}\text { Ponto de Quebra } \\
\qquad(0,45 ; 0,55)\end{array}$} & $P(y=1)<=0,45$ & 64 & 7 & 71 \\
\hline & $P(y=1)>0,55$ & 4 & 41 & 45 \\
\hline & Total & 68 & 48 & 116 \\
\hline & Corretos & 64 & 41 & 105 \\
\hline & $\%$ Corretos & 94,12 & 85,42 & 90,52 \\
\hline & $\%$ Incorretos & 5,88 & 14,58 & 9,48 \\
\hline \multirow{6}{*}{$\begin{array}{l}\text { Ponto de Quebra } \\
\qquad(0,40 ; 0,60)\end{array}$} & $P(y=1)<=0,40$ & 65 & 8 & 73 \\
\hline & $P(y=1)>0,60$ & 3 & 40 & 43 \\
\hline & Total & 68 & 48 & 116 \\
\hline & Corretos & 65 & 40 & 105 \\
\hline & $\%$ Corretos & 95,59 & 83,33 & 90,52 \\
\hline & $\%$ Incorretos & 4,41 & 16,67 & 9,48 \\
\hline
\end{tabular}

Tabela 3 - Capacidade de previsão do modelo estimado (pontos de quebra: $0,50 / 0,55 / 0,60$ ) 


\section{Egitania}

$s$ c i e $\Omega$ c i a

O modelo adotado fornece para cada empresa e para cada ano uma previsão da probabilidade com valores entre 0 e 1 . Os mais próximos de 1 correspondem às ações que irão ter rendibilidades supranormais positivas e os mais próximos de 0 às que irão ter as rendibilidades supranormais negativas. Neste contexto, definimos como pontos de quebra os valores de 0,50, 0,55 e 0,60. A título de exemplo, o ponto de quebra 0,60 significa que se prevê que as ações com rendibilidade supranormais positivas são as que têm uma probabilidade superior a 0,60 e as ações com rendibilidades supranormais negativas são as que têm uma probabilidade inferior ou igual a 0,40. Estes valores são comparados com os valores observados de $Y$ e são apresentadas as percentagens de respostas corretas e incorretas do modelo. As ações cujas probabilidades se situam entre 0,60 e 0,40 são eliminadas.

Analisando a tabela 3, concluímos que o modelo prevê corretamente 89,66\% das respostas no caso do ponto de quebra de 0,50 e 90,52\% no caso no ponto de quebra de 0,55 e 0,60. Os resultados indicam ainda que a percentagem de respostas incorretas do modelo é reduzida, traduzindo-se, no máximo, em 12 observações incorretamente classificadas. Por outro lado, verifica-se que a regressão se mostra capaz de prever com maior eficácia as ações que vão ter rendibilidades supranormais negativas do que as que vão ter rendibilidades supranormais positivas. De fato, o modelo prevê corretamente as rendibilidades supranormais negativas em $91,18 \%$ dos casos quando o ponto de quebra é de 0,50, 94,12\% quando é de 0,55 e 95,59\% quando é de 0,60. Quanto mais elevado o ponto de quebra, melhores resultados o modelo apresenta. Já para prever as rendibilidades supranormais positivas, a percentagem de respostas corretas é de 87,50\% no caso de 0,50, 85,42\% no caso de 0,55 e de 83,33\% no caso de 0,60. Neste caso, a eliminação das ações com probabilidades mais próximas de 0,50 não melhora os resultados do modelo.

Comparando os resultados obtidos com os dos estudos já existentes, é possível afirmar que o modelo agora estimado apresenta capacidades de previsão superiores. Por exemplo, no modelo usado no estudo de Ou e Penman (1989), a percentagem de previsões corretas era apenas de 60\% para ponto de quebra de 0,50 e de 66\% para um ponto de quebra de 0,60.

\section{RESULTADOS DAS ESTRATÉGIAS DE INVESTIMENTO}

As estratégias de investimento levadas a cabo a partir das previsões do modelo referido são construídas com base em dois métodos diferentes. A primeira estratégia de investimento assenta nas previsões do modelo tendo como base os indicadores financeiros relativos ao ano subsequente àqueles que foram utilizados na estimativa do modelo. Uma vez que na estimativa do modelo foram usados os dados referentes aos anos 2004-2007, nesta primeira estratégia serão utilizados os indicadores financeiros relativos ao ano de 2008. Partindo daí, serão previstas as rendibilidades médias supranormais para horizontes de investimento de 12, 24, 36 e 48 meses. Na segunda estratégia, as rendibilidades médias supranormais serão calculadas com base nas previsões do modelo ano após ano. Os indicadores financeiros, referentes aos anos de 2008 a 2011, serão introduzidos no modelo de forma a obter previsões para as rendibilidades médias supranormais para um horizonte de investimento de 12 meses. Nas duas 


\section{Eqitania}

$s$ c i e $\Omega$ c i a

sub-secções deste capítulo são apresentados e discutidos os resultados de cada uma das estratégias.

\subsection{RESULTADOS DA PRIMEIRA ESTRATÉGIA DE INVESTIMENTO}

A primeira estratégia de investimento assenta na previsão das ações que vão ter rendibilidades supranormais positivas e negativas tendo por base a informação das empresas para o ano de 2008, sendo que será assumida uma posição longa nas ações com rendibilidade supranormal positiva e uma posição curta nas ações com rendibilidade supranormal negativa. $\bigcirc$ resultado da estratégia consistirá na rendibilidade supranormal acumulada obtida durante diversos períodos de investimento (12, 24, 36 e 48 meses). Por exemplo, a rendibilidade obtida para o horizonte de 24 meses, será a que se obteria mantendo umas posições longa (curta) nas ações que, de acordo com o modelo e com os indicadores financeiros relativos ao ano de 2008, terão uma maior (menor) probabilidade de obter rendibilidades supranormais positivas entre maio de 2009 e abril de 2011 (período de 24 meses).

A probabilidade das ações de cada uma das 29 empresas terem uma rendibilidade supranormal positiva ou negativa é obtida através da equação (3) e a partir dos valores dos 49 indicadores financeiros relativos ao ano de 2008. Na formação das carteiras, as 29 ações são distribuídas em igual número por 10 carteiras diferentes (com exceção da carteira n. 5 que conta apenas com duas ações). Assim, as carteiras 1 e 10 são as que contêm as ações com maior probabilidade de rendibilidades supranormais positivas e negativas, respetivamente. Nas carteiras 1 a 3 será assumida uma posição longa e nas carteiras 8 a 10 será assumida uma posição curta. As carteiras intermédias 4 a 7 não são consideradas na estratégia de investimento.

As rendibilidades acumuladas para um horizonte de investimento de 12, 24, 36 e 48 meses são apresentadas na tabela 4, a seguir.

\begin{tabular}{|c|c|c|c|c|}
\cline { 2 - 5 } \multicolumn{1}{c|}{} & \multicolumn{4}{c|}{ Horizonte de investimento } \\
\cline { 2 - 5 } & 12 meses & 24 meses & 36 meses & 48 meses \\
\hline Carteira 1 & $-0,00820$ & $-0,01801$ & $-0,02481$ & $-0,03727$ \\
\hline Carteira 2 & $-0,00432$ & $-0,01038$ & $-0,01453$ & $-0,02434$ \\
\hline Carteira 3 & $-0,00348$ & $-0,02370$ & $-0,04304$ & $-0,04027$ \\
\hline Carteira 4 & 0,00695 & 0,00587 & 0,00827 & 0,01271 \\
\hline Carteira 5 & $-0,00005$ & $-0,00937$ & $-0,00295$ & $-0,02443$ \\
\hline Carteira 6 & $-0,01180$ & $-0,02799$ & $-0,02907$ & $-0,05784$ \\
\hline Carteira 7 & $-0,00471$ & $-0,00813$ & $-0,01066$ & $-0,00024$ \\
\hline Carteira 8 & 0,00566 & $-0,00552$ & $-0,00236$ & $-0,00962$ \\
\hline Carteira 9 & $-0,01611$ & $-0,02646$ & $-0,03305$ & $-0,02488$ \\
\hline Carteira 10 & 0,00472 & $-0,00961$ & $-0,01287$ & $-0,01338$ \\
\hline Posições longas (1 a 3) & $-0,00533$ & $-0,01736$ & $-0,02746$ & $-0,03396$ \\
\hline Posiç̃̃es curtas (8 a 10) & $-0,00191$ & $-0,01386$ & $-0,01609$ & $-0,01596$ \\
\hline Total de posições & $-0,00342$ & $-0,00350$ & $-0,01137$ & $-0,01800$ \\
\hline
\end{tabular}

Tabela 4 - Rendibilidades supranormais médias acumuladas de acordo com a previsão do modelo com dados de 2008 e para um horizonte de investimento de 12, 24, 36 e 48 meses 


\section{Egitania}

$s$ c i e $\Omega$ c i a

Constata-se que nenhuma das estratégias de investimento permite obter rendibilidades médias supranormais positivas. Apesar da estratégia de venda das ações, em todos os períodos de investimento analisados, ter gerado ganhos superiores à média do mercado, a estratégia de compra gerou sempre rendibilidades médias supranormais acumuladas negativas. Este diferencial levou a que a estratégia global não fosse bem-sucedida. Os resultados parecem confirmar que, como adiantado anteriormente, o modelo prevê melhor as empresas que irão ter rendibilidades supranormais negativas do que as que irão ter rendibilidades supranormais positivas.

Na tabela 5, a seguir, comparam-se os resultados agora obtidos com os apresentados por Holthausen e Larcker (1992), seguindo o mesmo procedimento.

\begin{tabular}{|c|c|c|c|c|c|}
\cline { 3 - 6 } \multicolumn{2}{c|}{} & \multicolumn{4}{c|}{ Horizonte de investimento } \\
\cline { 3 - 6 } & 12 meses & 24 meses & 36 meses & 48 meses \\
\hline \multirow{4}{*}{ Presente estudo } & Posições longas & $-0,00533$ & $-0,01736$ & $-0,02746$ & $-0,03396$ \\
\cline { 2 - 6 } & Posições curtas & $-0,00191$ & $-0,01386$ & $-0,01609$ & $-0,01596$ \\
\cline { 2 - 6 } & $\begin{array}{c}\text { Total de } \\
\text { posições }\end{array}$ & $-0,00342$ & $-0,00350$ & $-0,01137$ & $-0,01800$ \\
\hline \multirow{3}{*}{$\begin{array}{c}\text { Holthausen e } \\
\text { Larcker (1992) }\end{array}$} & Posiç̧̃es longas & 0,0003 & 0,0200 & 0,0416 & 0,0758 \\
\cline { 2 - 6 } & Posições curtas & $-0,0423$ & $-0,0558$ & $-0,0616$ & $-0,0399$ \\
\cline { 2 - 6 } & $\begin{array}{c}\text { Total de } \\
\text { posições }\end{array}$ & 0,0426 & 0,0758 & 0,1032 & 0,1157 \\
\hline
\end{tabular}

Tabela 5 - Rendibilidade supranormais médias acumuladas obtidas no presente estudo e no estudo de Holthausen e Larcker (1992)

Como se pode observar, a rendibilidade supranormal média acumulada obtida por Holthausen e Larcker (1992) é superior em todos os horizontes de investimento analisados. As diferenças são significativas, em desfavor da estratégia aplicada às ações portuguesas, tanto nas carteiras em que se assume uma posição longa como nas carteiras em que se assume uma posição curta. Os resultados sugerem que a informação financeira relativa a períodos de crescimento tem uma utilidade limitada para prever a evolução do mercado de ações em períodos de crise.

\subsection{RESULTADOS DA SEGUNDA ESTRATÉGIA DE INVESTIMENTO}

Na segunda estratégia de investimento são realizadas previsões, ano a ano. Assim, para cada ano entre 2008 a 2011, foram realizadas previsões e calculadas as rendibilidades médias supranormais para um horizonte de investimento de 12 meses. Por exemplo, os indicadores financeiros relativos ao ano de 2010 são utilizados no modelo estimado para se efetuarem previsões quanto à rendibilidade supranormal das ações no período que medeia entre maio de 2011 e abril de 2012.

Os valores previstos pelo modelo (com base na equação (3)) para o período de 2008 a 2011 permitiram definir as ações a adquirir e vender em cada ano. As rendibilidades acumuladas das carteiras anuais, formadas da forma descrita na secção anterior, foram calculadas para um 


\section{Egitania}

$s$ c i e $\Omega$ c i a

horizonte de investimento de 12 meses. Na tabela 6, a seguir, apresentam-se as rendibilidades supranormais médias obtidas em cada um dos anos estudados.

\begin{tabular}{|c|c|c|c|c|}
\cline { 2 - 5 } & \multicolumn{4}{|c|}{ Ano dos Dados Usados na Previsão } \\
\cline { 2 - 5 } & 2008 & 2009 & 2010 & 2011 \\
\hline Carteira 1 & $-0,00820$ & $-0,01725$ & $-0,00399$ & $-0,01900$ \\
\hline Carteira 2 & $-0,00432$ & $-0,00706$ & 0,00688 & 0,00242 \\
\hline Carteira 3 & $-0,00348$ & $-0,02414$ & $-0,01036$ & $-0,00147$ \\
\hline Carteira 4 & 0,00695 & $-0,01125$ & $-0,00341$ & $-0,00132$ \\
\hline Carteira 5 & $-0,00005$ & 0,00520 & 0,01828 & 0,00865 \\
\hline Carteira 6 & $-0,01180$ & $-0,00605$ & $-0,00282$ & 0,00423 \\
\hline Carteira 7 & $-0,00471$ & 0,00903 & 0,00207 & $-0,00523$ \\
\hline Carteira 8 & 0,00566 & $-0,00847$ & $-0,00716$ & $-0,02083$ \\
\hline Carteira 9 & $-0,01611$ & $-0,01592$ & $-0,00936$ & 0,00828 \\
\hline Carteira 10 & 0,00472 & $-0,02140$ & $-0,01362$ & $-0,02189$ \\
\hline Posições longas (1 a 3) & $-0,00533$ & $-0,01615$ & $-0,00249$ & $-0,00602$ \\
\hline Posições curtas (8 a 10) & $-0,00191$ & $-0,01527$ & $-0,01005$ & $-0,01148$ \\
\hline Total de posições & $-0,00342$ & $-0,01615$ & 0,00755 & 0,00546 \\
\hline
\end{tabular}

Tabela 6 - Rendibilidades supranormais médias acumuladas para um horizonte de investimento de 12 meses com dados referente aos anos de 2008 a 2011

total das posições revela que as perdas se verificam nos primeiro e segundo anos da estimativa. Nos restantes dois períodos, as rendibilidades supranormais médias acumuladas são positivas. Em todos os anos analisados, o investidor teria obtido ganhos nas posições curtas (estas revelam rendibilidades médias acumuladas negativas) e perdas nas posições longas. Este resultado confirma a conjetura de que o modelo tem maior capacidade de previsão das ações que terão rendibilidades negativas.

Também neste caso, os resultados encontrados estão aquém dos evidenciados para o mercado dos EUA por Holthausen e Larcker (1992). A rendibilidade anual média supranormal das para o período estudado por estes autores é de 2,7\% para as posições longas e de -4,6\% para as posições curtas. Já no presente estudo, as rendibilidades médias supranormais são de $-0,75 \%$ e de -0,97\%, respetivamente. Estes valores traduzem uma rendibilidade total de 7,3\% no caso de Holthausen e Larcker (1992) e de apenas 0,22\% no caso das ações transacionadas na Euronext Lisbon. Se tivermos em conta os custos associados às transações, facilmente concluímos que a rendibilidade da estratégia descrita será, em termos líquidos, negativa.

Os resultados da segunda estratégia de investimento corroboram os resultados obtidos com a primeira estratégia. Mais uma vez, e contrariamente aos resultados obtidos em estudos semelhantes, as rendibilidades evidenciadas permitem concluir que a informação financeira obtida em períodos de crescimento tem uma utilidade limitada na previsão das rendibilidades em períodos de crise. 


\section{Egitania \\ s c i e $\Omega$ c i a}

\section{CONCLUSÕES}

As estratégias fundamentais têm uma tradição importante nos mercados financeiros. A sua utilidade tem sido confirmada por vários autores ao longo dos últimos anos.

Neste artigo analisamos a utilidade da informação fundamental, representada por 49 indicadores financeiros, na previsão das rendibilidades das ações cotadas na Euronext Lisbon. O estudo decorre no contexto da crise financeira pós-2007.

Os resultados obtidos contrariam a generalidade da evidência empírica sobre o tema. No nosso estudo, a informação fundamental não permite conceber estratégias de investimento capazes de gerar rendibilidades superiores às obtidas por uma estratégia passiva. Embora as posições curtas das estratégias permitam obter, em todos os casos analisados, resultados supranormais positivos, as rendibilidades obtidas nas posições longas mais do que compensam essas rendibilidades. Isso significa que a análise fundamental se mostrou eficaz apenas na previsão das ações que irão gerar rendibilidades negativas.

Os resultados obtidos sugerem que o aumento da incerteza provocado por eventos como crises financeiras retira utilidade às estratégias baseadas no recurso a indicadores usados na análise fundamental. Esta interpretação é consistente com as evidências existentes acerca das diferenças de comportamento dos investidores em períodos de crise e em períodos tranquilos. A literatura sugere que os investidores se mostram mais sensíveis à informação em períodos negativos. A informação é, em geral, mais rapidamente incorporada nos preços em períodos de maior ansiedade e tensão pelo que é mais difícil utilizá-la em estratégias capazes de obter rendibilidades supranormais. Por exemplo, Cooper et al. (2004) concluem que a informação histórica dos preços só permite obter rendibilidades supranormais positivas (estratégias de momentum) em alturas em que o mercado regista uma tendência de subida. Peng et al. (2011) mostram que os investidores em fundos de investimento utilizam de forma mais cuidadosa a informação em alturas de sentimento negativo no mercado. E, num estudo mais recente, García (2013) verifica que os preços refletem muito mais rapidamente as notícias publicadas na secção financeira do jornal New York Times durante períodos de recessão.

Os resultados apresentados têm também implicações na perspetiva do investidor: em alturas de crise os investidores devem encarar com acrescido ceticismo as estratégias que mostraram ser lucrativas nos períodos que antecederam essas crises.

\section{REFERÊNCIAS}

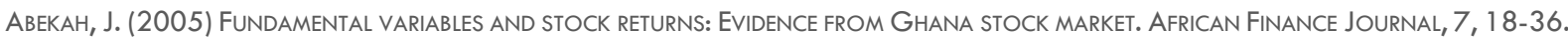

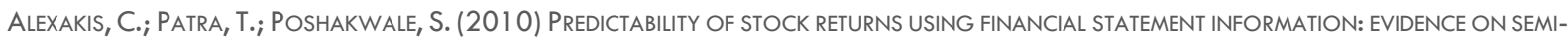
Strong efFiciency of EMERGING Greek Stock MARKet. Applied FinANCIAL ECONOMICS, 20, 1321-1326.

Avkiran, N.K.; Morita, H. (2010) Predicting Japanese bank stock Performance With a composite relative efficiency metric: A new INVESTMENT TOOL. PACIFIC-BASIN FINANCE JOURNAL, 18, 254-271. 


\section{Eqitania \\ s c i e $\cap$ c i a}

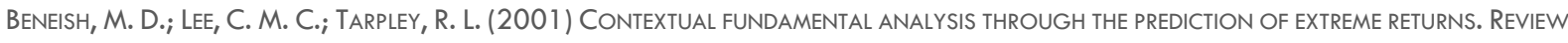
OF ACCOUNTING STUDIES, 6, 165-189.

BAKER, S. R.; BLOOM, N.; DAVIS, S. J. (201 2) MEASURING ECONOMIC POLICY UNCERTAINTY. WORKING PAPER.

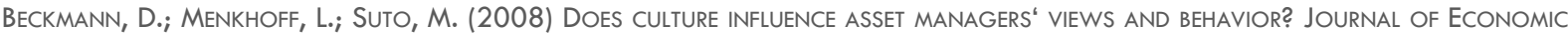
BEHAVIOR \& ORGANIZATION, 67, 624-643.

BlOOM, N. (2014) Fluctuations in UnCertainty. Journal of ECONOmic PerspeCtives, 28, 153-176.

Charitou, A.; Panagiotides, G. (1999) Financial analysis, future earnings and CASH Flows, and the prediction of Stock Returns: EVIDENCE FOR THE UK. ACCOUNTING AND BUSINESS RESEARCH, 29, 281-298.

Cheung J. K.; Chung, R.; Kim, J. B. (1997) The Profitability of Trading Strategies Based on Book Value and Earnings in Hong Kong:

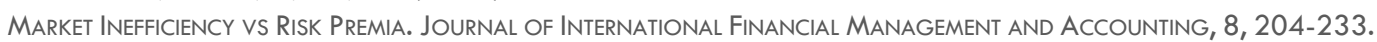

Chul, A.; Titman, S.; Wel, K. C. J. (2010) Individualism AND MOMENTUM AROUND the WORLD. Journal OF FinANCE, 65, 361 -392.

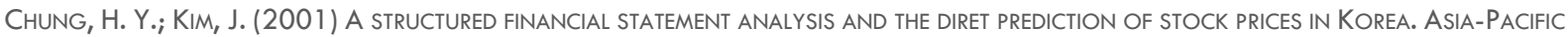
FINANCIAL MARKETS, 8, 87-117.

Cooper, M. J.; Gutierrez, JR., R. C.; Hameed, A. (2004) Market States and momentum. Journal Of Finance, 59, 1345-1365.

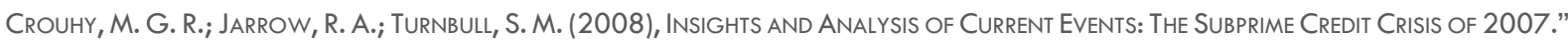
JoURNAL OF DERIVATIVES, 15, 81-110.

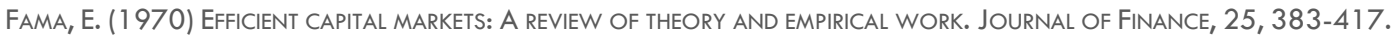

Frankel, R.; Lee, C. M. C. (1998) Accounting Valuation, market expectation, and Cross-sectional stock Returns. Journal OF ACCOUNTING AND ECONOMICS, 25, 283-319.

García, D. (2013) Sentiment during ReCessions. Journal OF FINANCE, 68, 1267-1300.

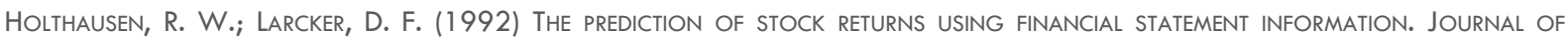
ACCOUNTING AND ECONOMICS, 15, 373-411.

Hyde, C.E. (2014) AN emerging markets analysis Of the Piotroski F sCore. JANSAA The Finsia Journal of Applied FinanCE, 2, 23-28.

Kahneman, D.; Slovic, P.; TVersky, A. (1982) Judgment Under UnCertainty, HeUristics and BiaSes Cambridge University Press.

KANG, J.; DING, D. (2005) VALUE AND GROWTH INVESTING IN ASIAN STOCK MARKETS 1991-2002. IN: CHEN, A. H. (COORD.), RESEARCH IN FINANCE, JAI PRESS, 1 $13-39$.

lobão, J.; Serra, A. P. (2007) Herding Behavior - Evidence from Portuguese Mutual Funds. In: Gregoriou, G. N. (Coord.), Diversification and Portfolio Management of Mutual Funds. Palgrave-Macmillan, 167-97.

Lobão, J.; Lopes, C. M. (2014) MOMentum Strategies in the Portuguese Stock Market. Aestimatio - The IEB International Journal Of FINANCE, 8, 68-89.

Martinez, I. (1999) Fundamental and Macroeconomic Information for the Security Prices Valuation: The French CaSe. Managerial FINANCE, 12, 17-30.

MOHANRAM, P. (2005) SEPARATING WINNERS FROM LOSERS AMONG LOW BOOK-TO-MARKET StOCKS USING FinANCIAL StATEMENT ANALYSIS. REVIEW OF ACCOUNTING STUdieS, 10, 133-170.

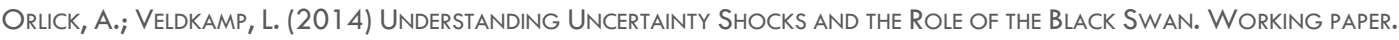




\section{Eqitania \\ s c i e $\Omega$ c i a}

Ou, J. A.; Penman, S. H. (1989) Financial statement analysis and the prediction of stock Returns. Journal of Accounting and ECONOMICS, 11, 295-329.

PenG, C. L.; Chen, M. L.; SHYU, S. D.; Wel, A. P. (2011) When IS MONEY LIKELY tO be SMART? EVIDENCE fROM MUtUAL FUND INVESTORS IN TAIWAN. INVESTMENT ANALYSTS JOURNAL, 73, 13-25.

Setiono, B.; Strong, N. (1998) Predicting stock Returns using financial statement information. Journal of Business Finance \& ACCOUNTING, 25, 631-657.

Shiller, R. (2000) IRRATIONAL EXuberance, Princeton University Press.

TANTIPANICHKUL, P. (2011) SEPARATING WINNERS FROM LOSERS IN THAI STOCK MARKETS USING FINANCIAL STATEMENT ANALYSIS, WORKING PAPER. 